\title{
Searching for primordial black hole evaporation signal with AMON
}

\section{Gordana Tešić*广}

The Pennsylvania State University

E-mail: gut10@psu. edu

\begin{abstract}
Primordial black holes (PBHs) are expected to gradually evaporate and then explode violently during the last few seconds of their lives, each one producing a burst of high energy particles. For PBHs evaporating near the Sun $(r \lesssim 1 \mathrm{pc}$ ), these particles could be detected in coincidence by several observatories with large fields of view, such as IceCube (neutrinos), HAWC (gamma rays) and Pierre Auger (gamma rays, neutrons and protons). The short temporal structure of the anticipated $\mathrm{PBH}$ evaporation signal provides a very low false positive rate for any possible detection. We will present the discovery potential of the Astrophysical Multimessenger Observatory Network $(\mathrm{AMON})$ for $\mathrm{PBH}$ evaporation events. AMON aims to discover multimessenger transient sources by performing real-time and archival coincidence searches from multiple observatory subthreshold data streams. In this approach, a distinctive $\mathrm{PBH}$ evaporation signature may be probed by conducting coincidence analysis from a few years of subthreshold neutrino, gamma-ray and cosmic ray data. Detection of PBH evaporation events would be a scientific breakthrough confirming Hawking's hypothesis of black hole radiation and cosmological models of phase transitions, and would allow us to probe physics at the highest energy scale as well as quantum gravity.
\end{abstract}

PACS: 95.85.Pw, 95.85.Ry, 04.70.Dy

The 34th International Cosmic Ray Conference,

30 July- 6 August, 2015

The Hague, The Netherlands

\footnotetext{
* Speaker.

${ }^{\dagger}$ The AMON team URL: http://amon.gravity.psu.edu/participants.shtml
} 


\section{Introduction}

Primordial Black Holes (PBHs) may have been created from the gravitational collapse of overdense regions in the early universe. The initial masses of PBHs are of the order of the particle horizon mass at the time when they were formed: $M_{\mathrm{H}}=c^{3} t / G[1,2]$. Since the exact time of their creation is unknown, the initial PBH masses could be anywhere within a range which starts at the Planck mass up to the masses of supermassive black holes. Hawking hypothesized in 1974 that black holes of mass $M$ radiate elementary particles with a black body spectrum due to the quantum-gravitational effects taking place in the vicinity of the black hole horizon [3]. The temperature of the black hole of mass $M$ is defined as $T_{\mathrm{BH}}=1 /(8 \pi G M)$, where $c=\hbar=k=1$. As the black hole evaporates via Hawking radiation, it loses mass at a rate $\mathrm{d} M / \mathrm{d} t=-\alpha(M) / G^{2} M^{2}$ [4], where the number of degrees of freedom (dof) of each radiated particle is included in the factor $\alpha(M)$. The parameter $\alpha(M)$ is an increasing function of the PBH temperature $T_{\mathrm{BH}}$, which also rises as the evaporation progresses. As soon as $T_{\mathrm{BH}}$ reaches the quantum-chromodynamics energy scale of $\Lambda_{\mathrm{QCD}} \gtrsim 200 \mathrm{MeV}$, free quark and gluons will be emitted and fragmented further into hadrons, photons and leptons [5]. The main contribution to the final spectrum of most leptons (neutrinos, electrons and muons) as well as photons is due to these decaying hadrons, rather than due to direct Hawking emission.

The lifetime $\tau$ of the non-charged and non-rotating black hole is determined by its initial mass $M$ as: $t_{\text {evap }} \approx G^{2} M^{3} / 3 \alpha(M)$. In the standard model of particle physics framework with 128 dof available at low mass, this means that if PBHs formed with the initial mass of $\sim 5 \cdot 10^{11} \mathrm{~kg}$, they would be in their final stage of evaporation at the present epoch. The final stage of PBH evaporation is an explosive process resulting in a burst of very-high-energy (VHE; E $>100 \mathrm{GeV}$ ) and ultra-high-energy (UHE; E $>100 \mathrm{TeV}$ ) particles, which could be seen by detectors on Earth.

The discovery of PBHs would be a scientific breakthrough that confirms the Hawking hypothesis and cosmological models of phase transitions. Further, it would allow us to probe particle physics processes at the highest energy scale, as well as quantum gravity. The fraction of the mass of dark matter that may have initially existed in the form of PBHs could be measured as well.

In this proceeding, we examine sensitivities for detection of neutrinos (the IceCube Neutrino Observatory), gamma rays (HAWC $\mathrm{TeV} \gamma$-ray Observatory) and cosmic rays (the Pierre Auger Cosmic Ray Observatory) produced by the explosions of PBHs, by using multi-messenger, multifacility approach within the Astrophysical Multimessenger Observatory Network (AMON) framework. For Pierre Auger, we assume that cosmic rays (CRs) detected include protons, neutrons and gamma rays. In addition, deflections of protons due to the extragalactic and galactic magnetic fields can be neglected for Pierre Auger, because this facility is sensitive to PBH evaporation events in the local volume $(r \lesssim 0.001 \mathrm{pc})$.

\section{The Astrophysical Multimessenger Observatory Network (AMON)}

AMON links the world's leading high-energy neutrino, cosmic-ray, gamma-ray and gravitational wave observatories by performing real-time and archival coincidence searches for multimessenger astrophysical sources from observatory subthreshold data streams. The resulting coincidences will be distributed to interested parties in the form of electronic alerts for real-time 


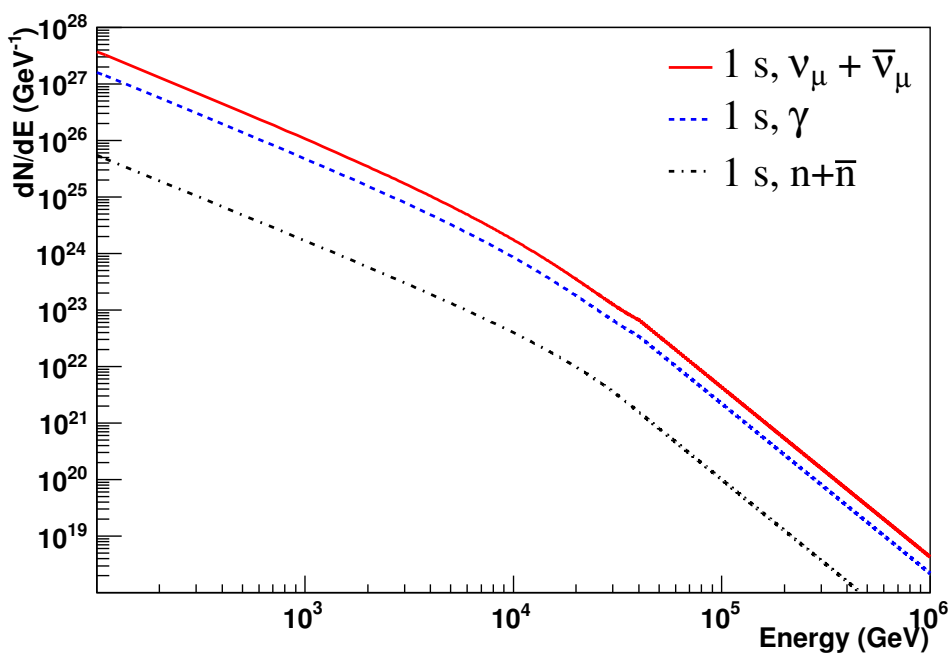

Figure 1: Time integrated PBH particle spectra for time window of $\Delta t=1$ second before complete evaporation: muon neutrinos (solid red), gamma rays (dashed blue) and neutrons/protons (dotted dashed black).

follow-up observation. The main goal of AMON is to probe the high-energy universe via all four fundamental forces of nature. Observatories that already signed the AMON Memorandum of Understanding (MoU) include: the IceCube and ANTARES Neutrino Observatories, the HAWC and VERITAS gamma-ray observatories, the Pierre Auger Cosmic Ray Observatory, the Swift satellite experiment and the MASTER robotic telescope network. More information about the AMON project can be found in Reference [6] and in the other AMON contribution to this conference [7].

\section{Sensitivities}

In this section we quantify the discovery potential of the AMON multi-messenger approach for detection of the individual PBH evaporation events. In particular, we examine a joint search for evidence of gamma-ray, neutrino and CR bursts due to evaporating PBHs in subthreshold data from the HAWC $\mathrm{TeV} \gamma$-ray observatory, the IceCube neutrino observatory and the Pierre Auger cosmic ray observatory. Subthreshold data, by definition, contain events of low significance and unrecoverable as astrophysical signals in the data stream of any individual facility. But if these low significance events are accompanied by an event from another multi-messenger channel, then jointly these events can achieve high significance, via coincidence analysis of the multiple data streams.

\subsection{Model predictions}

Here we consider the model of a non-rotating, uncharged black hole without a chromosphere by MacGibbon and Webber [5] for calculating the expected signal at each of these experiments. The possible presence of a PBH chromosphere $[8,9]$ would give a steeper particle spectra making these high-energy experiments not suitable for detection of the PBH bursts. The time-integrated particle spectra above $100 \mathrm{GeV}$ were calculated using methods from [5, 10-12]. The spectra are depicted in Figure 1. 


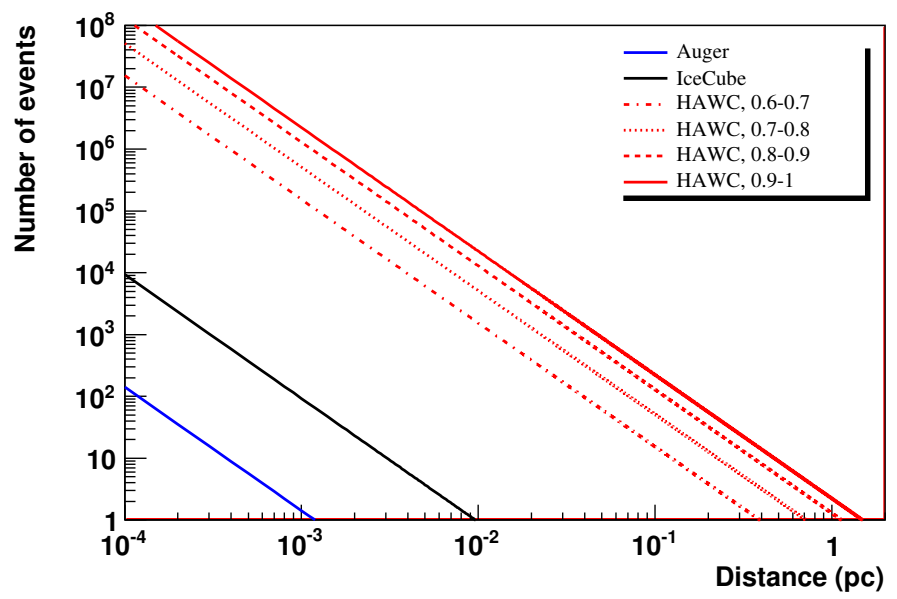

Figure 2: Expected number of events to be detected from an evaporating PBH during the last 5 seconds until complete evaporation as a function of distance: Pierre Auger (blue solid), IceCube (black solid) and four zenith angle bands for HAWC $(0.6<\cos \theta \leq 0.7$ - dotted dashed red, $0.7<\cos \theta \leq 0.8$ - dotted red, $0.8<\cos \theta \leq 0.9$ - dashed red and $0.9<\cos \theta \leq 1$ - solid red).

The predicted number of particles of type $\mathrm{X}$ (where $\mathrm{X}=$ neutrino, gamma-ray or neutron/proton) to be observed within a time window $\Delta t$ from a PBH at the distance $r$ from detectors at Earth, is given by:

$$
N_{\mathrm{X}}^{\mathrm{th}}=\frac{1}{4 \pi r^{2}} \int_{0}^{\Delta t} \int_{E_{\mathrm{D}}}^{\infty} \frac{\mathrm{d}^{2} N_{\mathrm{X}}(E, t)}{\mathrm{d} E \mathrm{~d} t} R(E, \theta) \mathrm{d} t \mathrm{~d} E,
$$

where $E_{\mathrm{D}}$ is the energy threshold for a given detector, $R(E, \theta)$ is the detector response function given as a function of zenith angle $\theta$ and particle energy, and $\mathrm{d}^{2} N_{\mathrm{X}}(E, t) / \mathrm{d} E \mathrm{~d} t$ is the particle $\mathrm{X}$ spectrum from the model. As shown in Figure 2, HAWC, IceCube and Pierre Auger observatories are sensitive to evaporating PBHs in the local region $(r<1.5 \mathrm{pc}$ for the most sensitive zenith angle band for HAWC).

The model predictions for HAWC were calculated by assuming background rate of $5.1 \mathrm{kHz}$ with the corresponding effective areas taken from Reference [13]. The muon neutrino effective area for the IceCube 86-string configuration was used [14]. Finally, for Pierre Auger, we assumed an effective area of $100 \mathrm{~km}^{2}$ in the energy range from 0.1 to $1 \mathrm{EeV}$, while the standard effective area of $3000 \mathrm{~km}^{2}$ [15] above $1 \mathrm{EeV}$ was used.

\subsection{Burst search strategy}

The signature burst at a given detector due to an individual PBH explosion consists of two or more particles (gamma rays, neutrinos and CR) arriving from the same direction from the sky and within a short time interval $\Delta t$. The spatial window to identify the burst is set to $3 \sigma$. The number of events belonging to a given burst defines the burst size. The total number of bursts of size $b$ that is expected to be observed over a period of observation $\tau$ is given by:

$$
n(b, \Delta t)=\rho_{\mathrm{PBH}} \tau V_{\mathrm{eff}}(b, \Delta t),
$$




\begin{tabular}{llll}
\hline \multicolumn{1}{c}{ Observatories } & \multicolumn{1}{c}{ Burst size } & $\Delta t(\mathrm{~s})$ & $V_{\mathrm{eff}}\left(\mathrm{pc}^{3}\right)$ \\
\hline \hline HAWC (alone) & $n_{\gamma} \geq 20$ & 5.0 & $1.4 \times 10^{-2}$ \\
IceCube (alone) & $n_{v} \geq 2$ & 5.0 & $3.4 \times 10^{-6}$ \\
Pierre Auger (alone) & $n_{c r} \geq 2$ & 0.1 & $3.5 \times 10^{-9}$ \\
HAWC \& IceCube & $n_{\gamma} \geq 19 \& n_{v} \geq 1$ & 10.0 & $2.2 \times 10^{-3}$ \\
HAWC \& Pierre Auger & $n_{\gamma} \geq 14 \& n_{c r} \geq 1$ & 5.0 & $2.1 \times 10^{-5}$ \\
IceCube \& Pierre Auger & $n_{v} \geq 1 \& n_{c r} \geq 1$ & 1.0 & $1.2 \times 10^{-6}$ \\
\hline
\end{tabular}

Table 1: The optimal time windows $(\Delta t)$ and corresponding effective volumes $\left(V_{\text {eff }}\right)$ for PBH searches. Values are shown for single experiment searches (IceCube, Pierre Auger and HAWC), as well as for joint searches for each pair of the experiments (HAWC-IceCube, HAWC-Pierre Auger and IceCube-Pierre Auger). The spatial window of $3 \sigma$ was used to define the bursts.

where $V_{\text {eff }}(b, \Delta t)$ is the effective volume of space probed by observations and $\rho_{\mathrm{PBH}}$ is the PBH density. The total effective volume $V_{\text {eff }}$ for all the bursts of sizes $b \geq b_{\text {th }}$ (where $b_{\text {th }}$ is the burst size threshold), can be expressed as:

$$
V_{\text {eff }}=\sum_{b=b_{\text {th }}}^{\infty} \int \mathrm{d} \Omega \int_{0}^{\infty} \mathrm{d} r r^{2} P\left(b, N_{\mathrm{X}}\right)
$$

where $P\left(b, N_{\mathrm{X}}\right)$ is the Poisson probability of detecting a burst of size $b$ assuming a model-predicted number of events $N_{\mathrm{X}}$ due to the particle X (gamma-ray, neutrino or neutron/proton). For multiple experiment searches (where $N$ is the number of experiments), one should replace the burst size $b$ by the vector $\vec{b}$ of each individual experiment's burst size $b_{i}$ with indices $i=1$ to $N$. In this case, the Poisson probability $P\left(b, N_{\mathrm{X}}\right)$ is replaced by the product $\prod_{i=1}^{N} P\left(b_{i}, N_{\mathrm{X}_{\mathrm{i}}}\right)$. More details on calculating the combined effective volume can be found in Reference [6].

A larger $\Delta t$ leads to more events detected within a given burst, thus increasing $V_{\text {eff. }}$ However, the larger time window also increases the number of background events. Consequently, this would require experiments to impose larger burst size thresholds $\vec{b}_{\text {th }}$ that would decrease $V_{\text {eff. }}$. The optimal search time window $\Delta t$ needs be chosen in such a way that it maximizes $V_{\text {eff }}$ while keeping the background rate low. Therefore, the optimization of $\Delta t$ needs to be performed separately for each observatory and for each combination of observatories.

Initially, a set of different $\Delta t$ ranging from 0.1 to 100 seconds was selected. Then, for each $\Delta t$, the event thresholds were chosen so that the false alarm rates (FAR) for burst detections were limited to $<1 \mathrm{yr}^{-1}$. Further, with these preselected thresholds $\vec{b}_{\text {th }}$, each effective volume was computed as a function of $\Delta t$. Finally, the optimal time windows were selected at those values for which $V_{\text {eff }}$ were found to be maximal. The optimal time windows and $V_{\text {eff }}$ are tabulated in Table 1 for each single observatory (HAWC, IceCube and Pierre Auger), as well as for the combined searches for any pair of these observatories (HAWC-IceCube, HAWC-Pierre Auger and IceCube-Pierre Auger).

\subsection{Anticipated detections and limits}

Several direct VHE searches for very short ( $\leq 30$ seconds) gamma-ray bursts due to the evapo- 


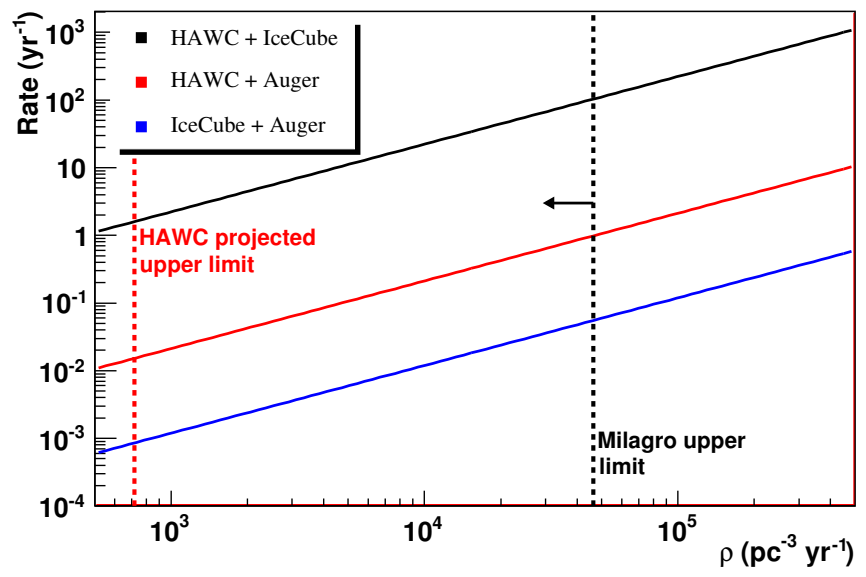

Figure 3: Expected number of joint bursts detection due to the evaporating PBHs: HAWC - IceCube (black), HAWC - Pierre Auger (red), and IceCube - Pierre Auger (blue). The current best upper limit (at 99.73\% CL) on PBH evaporation rate by Milagro (dashed black) as well as the expected limit from HAWC (dashed red) in case of no detection are shown. The HAWC limit was calculated assuming 2 transient bursts per year that are unrelated to PBHs were observed.

rating PBHs have been conducted (e.g., [16-19]) with no signal found. The current best upper limit on the number density of the evaporating PBHs $\left(\rho_{\mathrm{PBH}}\right)$ have been placed by the Milagro experiment [19] (also shown in Figure 3). It is expected that HAWC may give the best VHE constraint from a direct search on the $\rho_{\mathrm{PBH}}$ in case of a null PBH burst signal (Figure 3). However, HAWC may detect a gamma ray excess from an unknown transient source. In that case, it will be crucial to detect a multimessenger signal in order to distinguish between the $\mathrm{PBH}$ evaporation signature and other possible scenarios (GRBs or some other exotic type of transient burst). With just a few joint detections, a unique PBH temporal and spectral evaporation signature may be probed. In the parameter region currently not excluded by the best $\mathrm{PBH}$ upper limit of $\rho_{\mathrm{PBH}}<4.6 \times 10^{4} \mathrm{pc}^{-3} \mathrm{yr}^{-1}$ (at $99.73 \% \mathrm{CL}$ ), there is a possibility of 1-100 joint detections in the gamma-ray plus neutrino channel (HAWC and IceCube) within of one year of observation (see Figure 3). Due to the small effective volumes, joint detections in the other two channels (gamma-ray plus $\mathrm{CR}$ and neutrino plus $\mathrm{CR}$ ) are less likely, e.g., a few gamma-ray plus CR coincidences may be detected within $O(10)$ of years of joint observations by HAWC and Pierre Auger observatories.

In addition, this analysis is sensitive to various types of short-time scale bursts with a potential to discover other exotic transient sources, if they exist. For example, several models predict primordial relics with harder spectra (e.g., [20-26]) that may be detected by the highest-energy observatories such as IceCube and Pierre Auger.

\section{Conclusion}

We have examined the discovery potential for joint detections of multimessenger bursts due to evaporating PBHs. In our approach, using subthreshold data from the high-energy observatories 
such as HAWC, IceCube and Pierre Auger may lead to the discovery of the first PBH evaporation signature within one to a few years of observations. This multimessenger approach is essential to distinguish between bursts due to PBHs and other possible sources, should a positive detection occur. Within the AMON framework, both real-time and archival searches for PBH bursts are planned. This work is in progress.

\section{Acknowledgments}

This research is supported by grants from the U.S. National Science Foundation (003403953) and the Institute for Gravitation and the Cosmos of the Pennsylvania State University.

\section{References}

[1] Ya.B. Zel'dovich and I.D. Novikov, The Hypothesis of Cores Retarded during Expansion and the Hot Cosmological Model, Astron. Zh 43, 758 (1966) (Sov. Astron. 10, 602-3 (1967)).

[2] S.W. Hawking, Gravitationally collapsed objects of very low mass, Mon. Not. Roy. Astron. Soc. 152, 75-8 (1971).

[3] S.W. Hawking, Black hole explosions?, Nature 248, 30-1 (1974).

[4] D.N. Page, Particle emission rates from a black hole: Massless particles from an uncharged, nonrotating hole, Phys. Rev. D 13, 198-206 (1976).

[5] J.H. MacGibbon and B.R. Webber, Quark- and gluon-jet emission from primordial black holes: The instantaneous spectra, Phys. Rev. D 41, 3052-79 (1990).

[6] M.W.E. Smith et al., The Astrophysical Multimessenger Observatory Network (AMON) Astrop. Phys. 45, 56-70 (2013) [arXiv:1211.5602v1].

[7] G. Tešić (for the AMON team), AMON: transition to real-time operations, in proceedings of ICRC, POS ( ICRC2015) 329 (2015).

[8] A.F. Heckler, Calculation of the emergent spectrum and observation of primordial black holes, Phys. Rev. Lett. 78, 3430-33 (1997) [arXiv:astro-ph/9702027v1].

[9] R. Dighigh and J. Kapusta, High Temperature Matter and Gamma Ray Spectra from Microscopic Black Holes, Phys. Rev. D 65, 064028 (2002) [arXiv: gr-qc/0109090v1].

[10] J.H. MacGibbon, Quark- and gluon-jet emission from primordial black holes. II. The emission over the black-hole lifetime, Phys. Rev. D 44, 376-92 (1991).

[11] F. Halzen, E. Zas, J.H. MacGibbon and T.C. Weekes, Gamma rays and energetic particles from primordial black holes, Nature 353, 807-15 (1991).

[12] F. Halzen, B. Keszthelyi and E. Zas, Neutrinos from Primordial Black Holes, Phys. Rev. D 52 353, 3239-47 (1995) [arXiv: hep-ph/9502268v1].

[13] A.U. Abeysekara et al., On the sensitivity of the HAWC observatory to gamma-ray bursts, Astropart. Phys. 35, 641-650 (2012) [arXiv:1108.6034v2].

[14] M.G. Aartsen et al., Searches for Extended and Point-like Neutrino Sources with Four Years of IceCube Data ,ApJ 760, 109 (2014) [arXiv: 1406 . 6757].

[15] J. Abraham et al., Trigger and Aperture of the Surface Detector Array of the Pierre Auger Observatory, Nucl. Instrum. Methods A 613, 29-39, (2010) [arXiv:1111.6764]. 
[16] E.T. Linton et al., A new search for primordial black hole evaporations using the Whipple gamma-ray telescope, J. of Cosm. and Astropart. Phys. 1, 013 (2006).

[17] G. Tešić for the VERITAS Collaboration, Searching for primordial black holes with the VERITAS gamma-ray experiment, J. of Phys. Conf. Series 375, 052024 (2012).

[18] J-F. Glicenstein, A. Barnacka, M. Vivier, T. Herr, for the H.E.S.S. Collaboration, Limits on Primordial Black Hole evaporation with the H.E.S.S. array of Cherenkov telescopes, Proc. of the $33^{\text {st }}$ Int. Cosmic Ray Conf., (Rio de Janeiro) (2013) [arXiv:1307.4898v1].

[19] A.A. Abdo et al., Milagro Limits and HAWC Sensitivity for the Rate-Density of Evaporating Primordial Black Holes, Astrop. Phys. 64, 4-12, (2014) [arXiv: 1407 .1686v2].

[20] C.T. Hill, Monopolonium, Nucl. Phys. B 224, 469, (1983).

[21] R.J. Protheroe, P.A. Johnson, Propagation of Ultra High Energy Protons over Cosmological Distances and Implications for Topological Defect Models, Astropart. Phys. 4, 253, (1996) [arXiv:astro-ph/9506119].

[22] V. Berezinsky, M. Kachelrieß, A. Vilenkin, Ultra-high energy cosmic rays without GZK cutoff, Phys. Rev. Lett. 79, 4302, (1997), [arXiv: astro-ph/9708217].

[23] V. Berezinsky, P. Blasi, A. Vilenkin, Signatures of Topological Defects, Phys. Rev. D 58, 103515, (1998), [arXiv:astro-ph/9803271].

[24] S. Sarkar, R. Toldrà, The high energy cosmic ray spectrum from relic particle decay, Nucl. Phys. B, 621, 495, (2002), [arXiv: hep-ph/0108098].

[25] R. Aloisio, V. Berezinsky, M. Kachelrieß, On the status of superheavy dark matter, Phys. Rev. D, 74, 023516, (2006), [arXiv: astro-ph/0604311].

[26] J. Ellis, V.E. Mayes, D.V. Nanopoulos, UHECR Particle Spectra from Crypton Decays, Phys. Rev. D, 74, 115003, (2006), [arXiv:astro-ph/0512303]. 